\title{
Application of Imperialist Competitive Algorithm for Automated Classification of Remote Sensing Images
}

\author{
S. Karami and Sh.B. Shokouhi
}

\begin{abstract}
Recently, a novel evolutionary global search strategy called Imperialist Competitive Algorithm (ICA) has proven its superior capabilities in optimization problems.

This paper presents an application of ICA in automated clustering of remote sensing images. The proposed algorithm is basically a hierarchical two-phase process. At the first phase the original data set is decomposed into water bodies and land cover classes using near Infrared band's information. At the second phase, ICA has been applied to determine the number and centers of the land cover clusters using RGB band's information during an unsupervised clustering. The optimization is based on Fuzzy C-Means and an additional term for improving the accuracy of clustering. The method is applied on pan-sharpened IKONOS images of Tehran and 4 artificial data sets with different properties. Results obtained from applying the proposed method for both artificial data sets and RS image, indicate promising ability of this method in clustering data with unknown cluster number. Also the results show that the achieved overall accuracy can be available, better than $\mathbf{7 8 \%}$ in comparison with other applied methods.
\end{abstract}

Index Terms-Imperialist Competitive Algorithm, Remote Sensing, Hierarchical, Fuzzy C-Means Clustering

\section{INTRODUCTION}

The Remote Sensing (RS) technology is currently being offered a wide variety of digital imagery that covers most of earth's surface. Before the RS image data can yield the required information about the objects of interest, they need to be processed. The analysis and information extraction is the main part of the overall remote sensing process.

Classification of RS images is an important process that provides significant information for a variety of applications. Such information is a necessary input to many decision making and planning processes. Classification is the most common methodology in thematic map production from satellite images.

Supervised classification requires knowledge of the area at land. But in most of RS applications this knowledge is not sufficient available or the classes of interest are not yet defined. In these situations an unsupervised classification can be applied. Unsupervised classification of RS images eliminates the need for human operators to perform the time consuming and expensive process of training sample introduction. The art of unsupervised classification has been pursued by many researchers over the years and many different methods have been brought to bear on the subject.

Manuscript received February 17, 2012; revised March 30, 2012.

$\mathrm{S}$. Karami is with the corresponding author and can be contacted (e-mail: Karami_samaneh@yahoo.com).

S. B. Shokouhi is with the international scientific societies such as IEEE, IEICE and also Iranian Machine Vision and Image Processing (MVIP).
Clustering is an important unsupervised classification technique used in identifying some inherent structure present in a set of objects [1]-[2]. The purpose of cluster analysis is to classify objects into subsets that have some meaning in the context of a particular problem. More specifically, in clustering, a set of patterns, usually vectors in multi-dimensional space, are grouped into clusters in such a way that patterns in the same cluster are similar in some sense and patterns in different clusters are dissimilar in the same sense. In some clustering algorithms the number of classes that pixels must be grouped into is known a priori. In such situations clustering is formulated as distribution of pixels in $\mathrm{C}$ (number of classes) group, such that the pixels in a group are more similar to each other than the pixels in other groups. Most common classification algorithms such as K-Means and Fuzzy C-Means algorithms are applicable in such situations [3]. However, the major drawback of these clustering algorithms is that they often get stuck at local minima and the results are largely dependent on the choice of the initial center of classes. On the other hand, in most real life situations the number of clusters is not defined priori so the real challenge in this situation is to determine the number of optimal classes automatically.

In last several years, automated clustering has attracted a lot of research interests and many approaches have been proposed to determine the number of classes automatically. Genetic algorithm, one of the most popular optimization algorithms, has been applied for this purpose in many clustering problems [4]-[5].

In this paper we propose a new method that uses ICA to determine the number of optimal classes automatically and integrate the effectiveness of fuzzy C-Means algorithm, with the capability of ICA for providing the requisite perturbation to bring it out of the local minima.

ICA is a new optimization algorithm that has recently been introduced for solving different optimization problems. This algorithm is a global search strategy that uses the socio-political competition among empires as a source of inspiration [6]. ICA has been applied successfully in different domains namely designing controllers [7]-[8], recommender systems, characterization of elasto-plastic properties of materials [9] and many other optimization problems [10]-[11]. Comparing the results has shown great performance in both convergence rate and better global optima achievement. The effectiveness of the ICA based clustering technique is demonstrated on four artificial data sets having different characteristics. A real life application of clustering technique is provided for automatically clustering an IKONOS satellite image of a part of city Tehran into distinct regions. Then the performance of ICA in image clustering is compared with conventional GA and K-means 
clustering methods. The results obtained by the proposed algorithm and its comparison to GA and K-means indicate that the ICA has better performance in terms of both accuracy and convergence properties.

In most of the previous methods RGB properties of the different regions were used as similarity criteria for clustering data sets into different classes. One problem that reduces the clustering accuracy of RS images is that, due to suspended sediment and seaweeds present in the upper layers of the water body, these layers absorb more of the blue wavelengths and reflect the green, making the water appear greener in color. So in RS images water typically looks blue-green. Since water bodies and vegetation regions appear similar in color, RGB color property is not a comprehensive criterion to discriminate them. Comparing the spectral response of patterns we may be able to distinguish between targets, where we might not be able to, if we only compared them at RGB wavelengths. IR radiation is absorbed more by water than visible wavelengths, thus water and vegetation reflect somewhat similarly in the visible wavelengths but are always separable in the IR wavelength [12].

Because of this property of near infrared band, in proposed clustering approach, we use the near infrared Band of information to separate water body. Then RGB properties are used to determine the number of clusters and classification of land covers. In this regard, the proposed algorithm in this paper composes of two main steps. The first one separates water body and land cover regions and the second classifies land cover areas.

The remaining sections are organized as follows. Section II briefly introduces ICA and in section III, ICA is applied to determine the number of optimal clusters and clustering the $\mathrm{RS}$ images in 4-dimensional feature space during a hierarchical process. Section IV provides the experimental results. Eventually conclusion of the paper and remarks to the future are given in section $\mathrm{V}$.

\section{BRIEF DESCRIPTION OF ICA}

Imperialism is the policy of extending the power and rule of a government beyond its own boundaries. ICA is a novel global search strategy that uses imperialism and imperialistic competition process as a source of inspiration. This algorithm is based on that in real world countries try to extend their power over other countries in order to use their resources and bolster their own government. In fact, imperialist countries attempt to dominate other countries and turning them to their colonies. Also, imperialist countries compete strongly with each other for taking possession of other countries. During this competition stronger empires will get more power and the weakest one will ultimately collapse. This general policy of imperialist competition is used as the basis of the ICA. Fig. 1 shows the flowchart of this algorithm [6].

In optimization problems the final goal is to find an optimal solution of the variables of the problem. So in these problems an array of variables is formed to be optimized. In ICA, the term country is used for this array. In an $\mathrm{N}$-dimensional optimization problem, a country is an

$1 \times N$ array. The variable values of the country are floating point numbers.

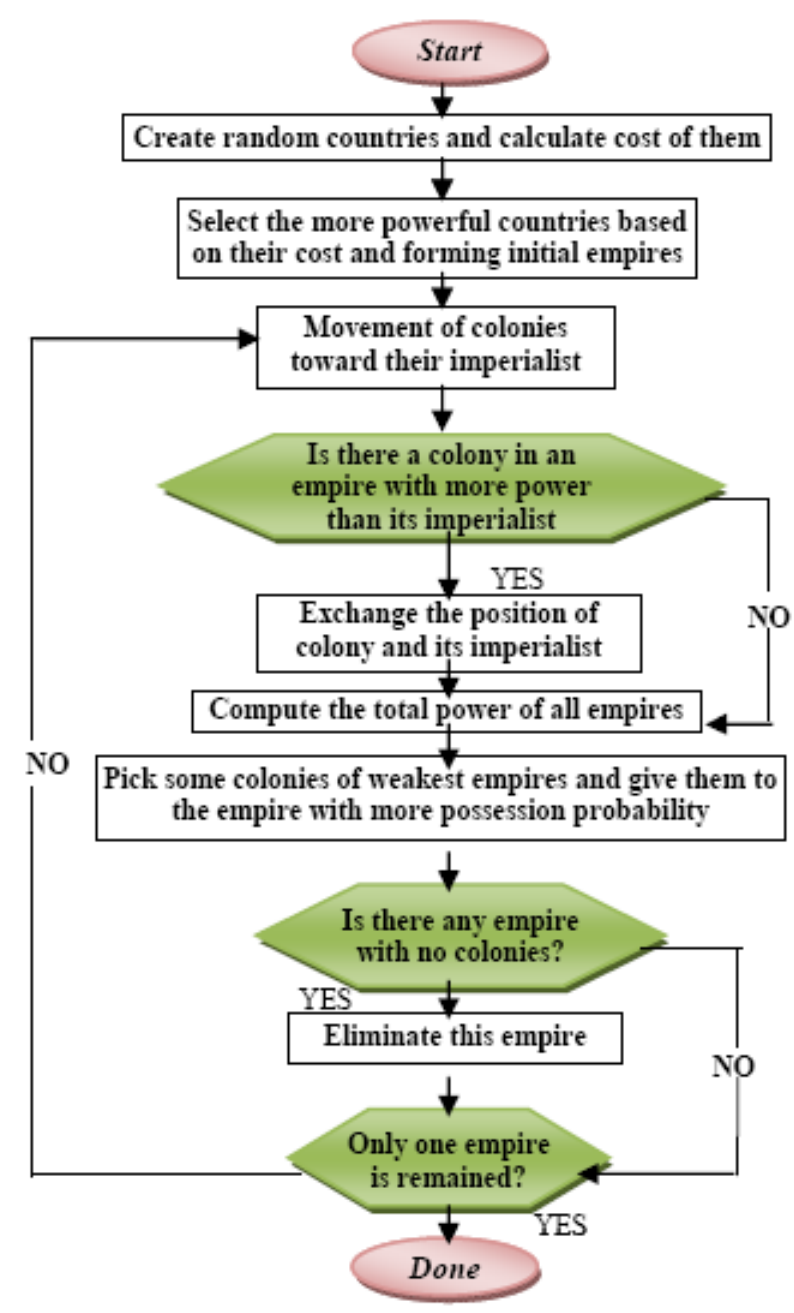

Fig. 1. Flowchart of the imperialist competitive algorithm.

As shown in Fig. 1, algorithm starts with some initial countries that are randomly dispread in search space. Some of the stronger countries (countries with lower cost) in the population are selected to be the imperialists and all the other countries are divided among them based on their power. The initial number of colonies of each imperialist should be directly proportionate to its normalized power. The normalized power of an imperialist is defined by

$$
P_{n}=\left|\frac{C_{n}}{\sum_{i=1}^{N_{i m p}} C_{i}}\right|
$$

where $C_{n}$ is the normalized cost of $n^{\text {th }}$ imperialist and is defined as difference between the cost of imperialist and total cost of all imperialists. Then the initial number of colonies of $n^{\text {th }}$ empire will be:

$$
N_{n}=\operatorname{round}\left\{P_{n} \cdot N_{c o l}\right\},
$$

where $N_{c o l}$ is the number of all colonies.

To form initial empires, each imperialist randomly picks $N_{n}$ of the colonies. After creating the initial empires, colonies start moving toward their relevant imperialist state. This process is called assimilation policy. In assimilation, each colony moves on the line that connects the colony and 
its imperialist by $\mathrm{x}$ units, which $\mathrm{x}$ is a random variable with uniform distribution. Then for $\mathrm{x}$ we have

$$
x \sim U(0, \beta d)
$$

where $\beta$ is a positive number which is considered to be smaller than 2 and $\mathrm{d}$ is the distance between the colony and its imperialist.

To create new positions for colonies around the imperialist in different directions, a random deviation angle $(\theta)$ is added to the direction of movement,

$$
\theta \sim U(-\alpha, \alpha)
$$

where $\alpha$ adjusts the deviation from original direction.

In fact $\beta$ and $\alpha$ are arbitrary numbers that modify the area that colonies randomly search around their corresponding imperialist.

If this movement causes to find a colony with better position (lower cost) than that of imperialist, the colony moves to the position of the imperialist and vice versa. The total power of an empire is defined by the power of imperialist state plus a percent of the mean power of its colonies.In imperialistic competition, all imperialists try to take the possession of other imperialist's colonies and increase their own power. In ICA, this imperialistic competition is modeled as simply taking some of the weakest colonies of the weakest empire and giving them to the empire that has the most probability of possessing them. The possession probability of each empire depends on its total power, so stronger imperialists will be more likely to possess selected colonies. The total power of an empire is mainly affected by the power of imperialist. But the power of the colonies has an effect, albeit negligible, on the total power. The total power of an empire is defined by

$$
P_{t}=P_{i m}+\text { ameam }\{\text { power }(\text { colonies })\},
$$

where $P_{i m}$ is the power of the imperialist and $\varepsilon$ is a positive number which is considered to be smaller than 1 .

As a result of imperialistic competition, the colonies of powerless empires will be divided among other imperialists and the empire will be collapsed. Collapse mechanism will cause an increase in the power of great empires and eliminating the weaker ones. As a result, all the countries converge to a state in which there exists only one empire that possesses all other countries as its colonies. Imperialistic competition and the movement of colonies toward their relevant imperialist will hopefully cause to make a new world with one empire that all its colonies have the same position and power as the imperialist. In this situation the imperialist consists of an array of variables which is an optimal solution of the problem. In addition to this stopping condition, in ICA like other evolutionary algorithms reaching a maximum number of iterations (decades) or having no improvement in final results for several consecutive iterations may be chosen as stopping criteria. In these situations, the solution of problem is determined by the imperialist of the most powerful empire. We applied ICA to find optimal cluster centers of an image during the hierarchical process based on fuzzy C-Means clustering analysis and an additional parameter for improving the accuracy of clustering.

\section{HieRARCHICAL IMAGE CLUSTERING With UNKNOWN Cluster Number USING ICA}

In this section, an attempt has been made to use ICA for automatic clustering a satellite image in 4-dimensional feature space with unknown cluster numbers.

As mentioned in section I, the clustering method proposed here mainly composes of two steps. The first one uses near IR band of information to separate water bodies and decomposing image into water and land cover regions. In the second one, we use ICA to determine the optimal number of clusters and proportional centers and classification of land cover regions based on RGB properties of image pixels. Each stage of this algorithm will be described subsequently.

\section{A. Decomposing Image into Water Body and Land Cover Classes Using ICA}

As described before, at the first step of proposed method, the grey level values of near Infra Red band is used as a similarity criterion for separating water bodies and land covers. So, two cluster centers should be determined. In this method we use ICA for determining the appropriate cluster centers and dividing all image pixels among the mentioned cluster centers based on their near IR band properties. As mentioned in section II, ICA starts with some initial countries that are randomly dispread in search space. Here the countries are arrays of two real numbers which each of them represents the near infra red gray level of one of the water and land cover cluster centers.

After initializing countries, the partition matrices of each country $U$ and the fuzzy cluster centers $Z_{i}$ are calculated by using

$$
u_{i j}=\left[\frac{\left|\tau i^{-\tau} j\right|}{\left|\tau_{1}-\tau j\right|}+\frac{\left|\tau_{i}-\tau j\right|}{\left|\tau 2^{-\tau} j\right|}\right]^{-1} \quad \text { for } 1 \leq i \leq \text { Cand } 1 \leq j \leq n
$$

Which $u_{i j}$ denotes the grade of membership of the $j^{t h}$ pixel to the $i^{t h}$ cluster, $n$ is the total number of image pixels, $\tau_{i}$ is the gray level value of $i^{t h}$ cluster center and $\tau_{j}$ is the gray level value of $j^{\text {th }}$ image pixel.

$$
Z_{i}=\frac{\sum_{j=1}^{n}\left(u_{i j}\right) \tau_{j}}{\sum_{j=1}^{n}\left(u_{i j}\right)} \quad \text { for } i=1,2
$$

Which $n$, is the total number of image pixels, ${ }^{\tau_{i}}$ is the gray level value of $i^{\text {th }}$ cluster center and $\tau_{j}$ is the gray level value of $j^{\text {th }}$ image pixel.

Then we calculate $U^{\text {new }}$ using $Z^{\text {old }}$ and $Z^{\text {new }}$ using new obtained $U$. We repeat this process until there is no more change in $Z$ for a fixed tolerance. 
After calculating cluster centers, we determine a crisp cluster structure by replacing the highest membership value of each pixel by 1 and allocating it to the related cluster. Using this method helps us to provide more information when the clusters present in the data are overlapping in nature. After forming clusters, the power of each country is calculated by using following equation:

$$
P(z, x)=\frac{\Delta(z)}{\sigma(z, \tau)}=\frac{\left|z_{1}-z_{2}\right|}{\sum_{i=1}^{n_{1}}\left|z_{1}-\tau_{i}\right|+\sum_{i=1}^{n_{2}}\left|z_{2}-\tau_{i}\right|}
$$

Which $\boldsymbol{n}_{\boldsymbol{i}}$ is the number of pixels belongs to the $i^{\text {th }}$ cluster.

After calculating the power of all countries, some of the best countries are selected as imperialists and all the other countries are divided among the mentioned imperialist based on their power. As mentioned in section 2, after creating initial empires colonies start moving toward their imperialists (In our implementation $\beta$ is a random number ranging from 0 to 2 and $\alpha$ is less than $0.5(\mathrm{rad}))$.

For all new positions we should use fuzzy C-Means clustering to form new clusters.

If the movement creates colonies with lower cost than their imperialists, the position of that colonies and imperialists will exchange. Then the total power of all empires is calculated and imperialistic competition start between empires. This process continues until one of the mentioned stopping conditions will be reached. In this situation the selected imperialist consists of an array of two variables which each of them represents gray level of one of the water and land cover cluster centers.

\section{B. Clustering Land Covers with Unknown Cluster Number Using ICA}

After separating water bodies, land covers should be clustered. The real challenge in this situation is to be able to automatically evolve an optimal value of cluster numbers as well as providing the appropriate clustering of land covers. In this section an attempt has been made to propose an algorithm that uses ICA for automatic clustering land covers. In this step countries are directly characterized as a real code string. The string is an array of 3-dimensional vectors, which encodes the centers of clusters in the RGB color space. Each string represents different number of cluster centers in 3 dimensions. Then if a country encodes $C_{i}$ cluster centers, the length of the string is $l=3 \times C_{i}$. The values of elements of each cluster center vector ranging from 0 to 255 .

After initializing countries, we calculate partition matrix $U$ for each country by using (9) as below

$$
u_{i j}=\left[\sum_{k=1}^{C}\left(\frac{D^{2}\left(c_{i}, x_{j}\right)}{D^{2}\left(c_{k}, x_{j}\right)}\right)\right]^{-1} \text { for } 1 \leq i \leq \text { Cand } 1 \leq j \leq n(9)
$$

Which $c_{i}$ is $3 \mathrm{D}$ vector of $i^{t h}$ cluster center, $D^{2}\left(c_{i}, x_{j}\right)$ is the Euclidean distance between $i^{\text {th }}$ cluster center and $j^{\text {th }}$ image pixel and $\mathrm{C}$ is the number of cluster centers of each country. Then we use (7) and repeat process until there is no more change in cluster centers. In such a condition as mentioned before pixels are divided among the cluster centers based on their membership grade and crisp clusters are formed.

After forming initial clusters, the empires are formed based on the power of countries.

The power of each country is defined by

$$
P(c, x)=\frac{\Delta(c)}{\sigma(c, x)}=\frac{\min D_{i \neq j}^{2}\left(c_{i}, c_{j}\right)}{\sum_{i=1}^{n_{i}} \sum_{j=1}^{C} D^{2}\left(x_{i}, c_{j}\right)}
$$

Which $p$ is the power of country, $n_{i}$ is the number of pixels belong to $i^{\text {th }}$ cluster, $c_{i}$ is $3 \mathrm{D}$ vector of $i^{t h}$ cluster center, $D^{2}\left(c_{i}, c_{j}\right)$ is the Euclidean distance between $i^{\text {th }}$ and $j^{t h}$ cluster centers and $D^{2}\left(x_{i}, c_{j}\right)$ is the Euclidean distance between $i^{\text {th }}$ pixel and $j^{\text {th }}$ cluster center.

Because of different lengths of countries the assimilation process is different from what mentioned in sec 2 . In this situation each 3D vector (Cluster center) of a colony randomly selects one of the vectors of its imperialist and moves toward it on the line that connects these two vectors with a small deviation angle. Then algorithm continues as described in last section until the stopping condition (variation in the power of best imperialist during 5 decades be less than a predefined threshold) will be reached. In this situation the selected imperialist consists of an array of $3 \mathrm{D}$ vectors which each of them represents a cluster center.

\section{IMPLEMENTATION RESULTS}

In this section, we present the results of our research.

In ICA the initial number of countries is set to 300 that 20 of them are chosen as initial imperialists to form initial clusters.

Also $\beta$ and $\alpha$ are set to 1.5 and 0.5 (rad) empirically.

The experimental results are provided for four 3D artificial data sets (Data1, Data2, Data3, Data4), with different properties and a satellite image of a part of city Tehran. Also a genetic based clustering algorithm (with the same cost function used in ICA) and K-means clustering algorithm are applied to cluster the satellite image.

Comparing the results obtained by these three methods shows the capability of our proposed hierarchical ICA based clustering algorithm in clustering data with unknown cluster numbers and overlapped clusters.

\section{A. Results on Artificial Data Sets}

The artificial data sets are generated using a Gaussian distribution in 3dimensional feature space, so for clustering, we utilize the second part of algorithm uses RGB properties of objects.

Data1 contains 4 clusters which are fully separate from each other. Data2 contains 4 clusters where two of them are overlapped. Data3 contains 4 clusters where are fully enclosed two by two. Data4 have 4 clusters where 3 of them 
are overlapped. Figs. 2-5 show the four data sets and tables I-IV show the confusion matrices of them, respectively.

From the results it can be seen that ICA in conjunction with improved fuzzy clustering method has successfully found exact number of clusters and clustered them with high accuracy in all four cases.

\section{B. Results of IKONOS Image Hierarchical Clustering}

In this section the utility of proposed clustering algorithm for partitioning pixels into different regions in satellite image, is investigated. IKONOS satellite image of a part of city Tehran in RGB bands is shown in Fig. 6. Fig. 7 shows, the clustered image using ICA based method and Fig. 8 illustrated a reference map which has been clustered manually. The land cover considered contains various kinds of soil which are different in color. Two clusters are distinguished by the algorithm as open space, which are shown in light and dark brown in Fig. 7. In confusion matrix and manually clustered image theses two regions are considered together as a single cluster. For evaluating the results all data points are compared with the reference map. The comparative result is shown in table V. Comparing the classified image with the reference map and also from the available ground truth, it is evident that ICA has indicated its capability in clustering images with unknown cluster numbers.

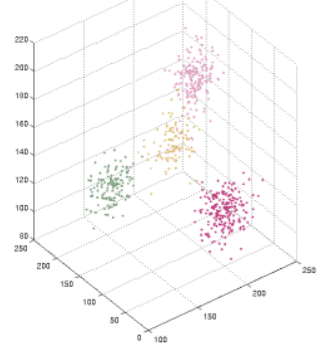

(a)

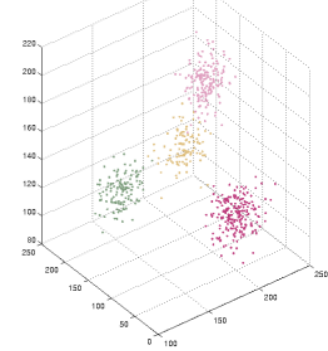

(b)
Fig. 2. (a) Data set1; (b) clustered data1

TABLE I: CONFUSION MATRIX FOR RESULTS OF FIG. 2

\begin{tabular}{|c|c|c|c|c|c|}
\hline \multirow{6}{*}{ 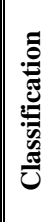 } & & \multicolumn{4}{|c|}{ Reference data } \\
\hline & & Cluster 1 & Cluster2 & Cluster3 & Cluster4 \\
\hline & Cluster I & 102 & 0 & 3 & 0 \\
\hline & Cluster2 & 0 & 208 & 0 & 0 \\
\hline & Cluster3 & 1 & 0 & 173 & 0 \\
\hline & Cluster4 & 0 & 0 & 0 & 132 \\
\hline \multicolumn{3}{|c|}{ Producer's accuracy } & \multicolumn{3}{|c|}{ User's accuracy } \\
\hline \multicolumn{3}{|c|}{ Cluster1: $99.02 \%$} & \multicolumn{3}{|c|}{ Cluster1: $97.14 \%$} \\
\hline \multicolumn{3}{|c|}{ Cluster2: $100 \%$} & \multicolumn{3}{|c|}{ Cluster2: $100 \%$} \\
\hline \multicolumn{3}{|c|}{ Cluster3: $98.29 \%$} & \multicolumn{3}{|c|}{ Cluster 3: $99.42 \%$} \\
\hline \multicolumn{3}{|c|}{ Cluster4: $100 \%$} & \multicolumn{3}{|c|}{ Cluster4: $100 \%$} \\
\hline
\end{tabular}

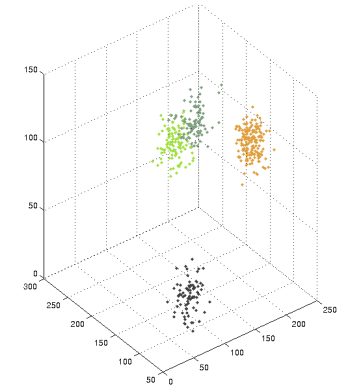

(a)

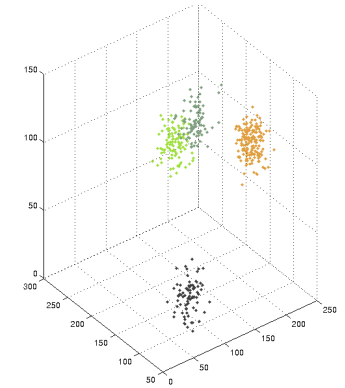

(b)
Fig. 3. (a) Data set2; (b) clustered data2
TABLE II: CONFUSION MATRIX FOR RESULTS OF FIG. 3

\begin{tabular}{|c|c|c|c|c|c|}
\hline \multirow{6}{*}{ 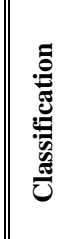 } & & \multicolumn{4}{|c|}{ Reference data } \\
\hline & & Cluster 1 & Cluster2 & Cluster 3 & Cluster4 \\
\hline & Cluster1 & 164 & 0 & 0 & 0 \\
\hline & Cluster2 & 0 & 84 & 0 & 12 \\
\hline & Cluster3 & 0 & 0 & 80 & 0 \\
\hline & Cluster4 & 0 & 7 & 0 & 93 \\
\hline \multicolumn{3}{|c|}{ Producer's accuracy } & \multicolumn{3}{|c|}{ User's accuracy } \\
\hline \multicolumn{3}{|c|}{ Cluster1: $100 \%$} & \multicolumn{3}{|c|}{ Cluster1: $100 \%$} \\
\hline \multicolumn{3}{|c|}{ Cluster2: $91.30 \%$} & \multicolumn{3}{|c|}{ Cluster2: $87.5 \%$} \\
\hline \multicolumn{3}{|c|}{ Cluster3: $100 \%$} & \multicolumn{3}{|c|}{ Cluster 3: $100 \%$} \\
\hline \multicolumn{3}{|c|}{ Cluster4:88.57\% } & \multicolumn{3}{|c|}{ Cluster4: $93.00 \%$} \\
\hline
\end{tabular}

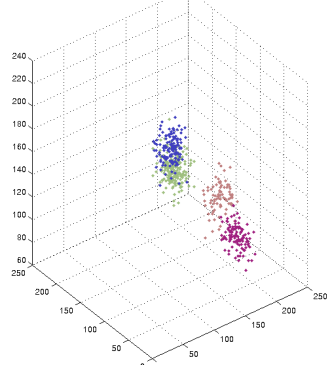

(a)

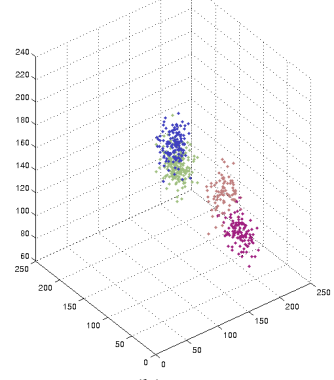

(b)
Fig. 4. (a) Data set3; (b) clustered data3

TABLE III: CONFUSION MATRIX FOR RESULTS OF FIG. 4

\begin{tabular}{|c|c|c|c|c|c|}
\hline \multirow{6}{*}{ 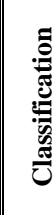 } & & \multicolumn{4}{|c|}{ Reference data } \\
\hline & & ClusterI & \multirow{2}{*}{ Cluster 2} & \multirow{2}{*}{$\frac{\text { Cluster3 }}{0}$} & \multirow{2}{*}{$\frac{\text { Cluster4 }}{0}$} \\
\hline & Cluster1 & 80 & & & \\
\hline & Cluster2 & 7 & 106 & 0 & 0 \\
\hline & Cluster3 & 0 & 0 & 124 & 14 \\
\hline & Cluster4 & 0 & 0 & 23 & 174 \\
\hline \multicolumn{3}{|c|}{ Producer's accuracy } & \multicolumn{3}{|c|}{ User's accuracy } \\
\hline \multirow{4}{*}{\multicolumn{3}{|c|}{$\begin{array}{l}\text { Cluster1: } 91.95 \% \\
\text { Cluster2: } 96.36 \% \\
\text { Cluster3: } 84.35 \% \\
\text { Cluster4: } 92.55 \%\end{array}$}} & \multicolumn{3}{|c|}{ Cluster1: $95.23 \%$} \\
\hline & & & \multicolumn{3}{|c|}{ Cluster2: $93.80 \%$} \\
\hline & & & \multicolumn{3}{|c|}{ Cluster3: $89.85 \%$} \\
\hline & & & Cluster4: & $88.32 \%$ & \\
\hline \multicolumn{6}{|c|}{ Overall accuracy: $\mathbf{9 0 . 9 7 \%}$} \\
\hline
\end{tabular}

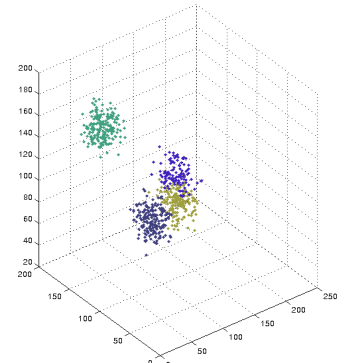

(a)

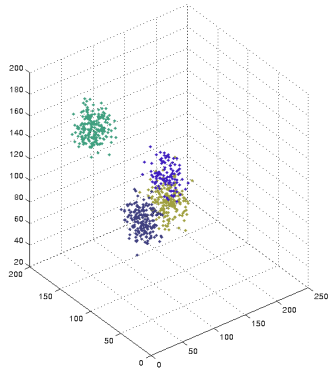

(b)
Fig. 5. (a) Data set4; (b) clustered data4

TABLE IV: CONFUSION MATRIX FOR RESULTS OF FIG. 5

\begin{tabular}{|c|c|c|c|c|c|}
\hline \multirow{6}{*}{ 氖 } & \multicolumn{5}{|c|}{ Reference data } \\
\hline & \multicolumn{2}{|c|}{ Cluster1 } & Cluster 2 & Cluster3 & Cluster4 \\
\hline & Cluster1 & 184 & 0 & 7 & 6 \\
\hline & Cluster2 & 0 & 204 & 0 & 0 \\
\hline & Cluster3 & 6 & 0 & 96 & 4 \\
\hline & Cluster4 & 3 & 0 & 5 & 178 \\
\hline \multicolumn{3}{|c|}{ Producer's accuracy } & \multicolumn{3}{|c|}{ User's accuracy } \\
\hline \multicolumn{3}{|c|}{\begin{tabular}{|l} 
Cluster1: $95.33 \%$ \\
Cluster2: $100 \%$ \\
Cluster3: $88.88 \%$ \\
Cluster4: $94.68 \%$
\end{tabular}} & \multicolumn{3}{|c|}{$\begin{array}{l}\text { Cluster1: } 93.40 \% \\
\text { Cluster } 2: 100 \% \\
\text { Cluster3: } 90.56 \% \\
\text { Cluster4: } 95.69 \%\end{array}$} \\
\hline
\end{tabular}




\section{Comparing the Performance of ICA, GA and K-Means Clustering Algorithms}

In this section the experimental results comparing the ICA-based clustering algorithm with the GA and K-means clustering algorithms are provided for IKONOS image.

According to the different clusters that can be specified in IKONOS image (Fig. 6), the value of $\mathrm{K}$ is chosen to be 5 and 6 in K-means clustering algorithm.

In GA the initial number of population, mutation and selection rates are set to $300,30 \%$ and $40 \%$ respectively and the number of clusters is unknown (as in ICA). The termination criterion for all three algorithms is set to reaching a maximum number of iterations or having no improvement in final results for several consecutive iterations (in ICA, countries converge to a state which there exists only one empire that possesses all other countries). Fig. 9(a) shows the clustered image of implementation GA based clustering algorithm. As shown in this figure, 6 clusters are detected by GA-clustering algorithm (vegetation, water, road, habitation and 2 clusters for soil). So the variety and number of optimal clusters, determined by GA is the same as ICA. Fig. 9(b) and Fig. 9(c) depict the results of K-means clustering algorithm with 5 and 6 classes as priori information. As it derived from the results when the number of initial clusters is set to 5, $\mathrm{K}$-means combines habitation, road and a part of soil classes and clusters them as a single class. Likewise, when the number of initial classes is 6 , this algorithm clusters road and habitation classes as a single class and classify soil into 3 classes. In fact, in this case K-means clustering algorithm is unable to provide meaningful clusters. Table VI shows the comparative results of three clustering algorithms considered in this paper. This is clear that, the user's and producer's accuracy of K-means clustering algorithm cannot be calculated because of not meaningful classes formed by this method (combining road, soil and habitation classes). Regarding table VI, it can be concluded that evolutionary algorithms can successfully classify data with unknown clusters and close RGB properties and they both perform better than K-means clustering algorithm. Also, comparison between ICA and GA indicates that ICA excels GA.

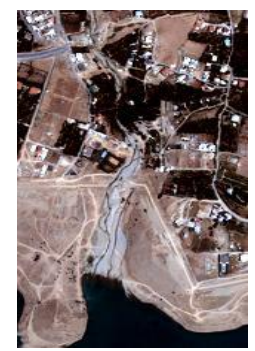

Fig. 6. IKONOS image of Tehran, in RGB bands

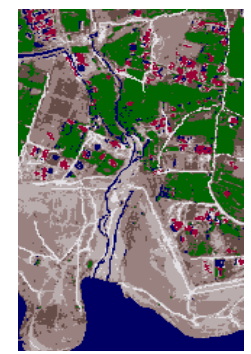

Fig. 7. Clustered image using the ICA based method

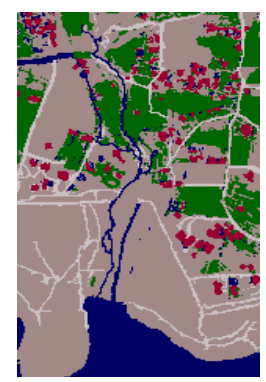

Fig. 9. Reference map clustered manually

TABLE V: CONFUSION MATRIX FOR RESULTS OF FIG. 7

\begin{tabular}{|c|c|c|c|c|c|c|}
\hline \multirow{7}{*}{ 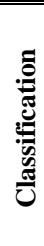 } & \multirow{7}{*}{$\begin{array}{l}\text { soil } \\
\text { Vegetation } \\
\text { road } \\
\text { water } \\
\text { habitation }\end{array}$} & \multicolumn{5}{|c|}{ Reference map } \\
\hline & & soil & Vegetation & road & water & habitation \\
\hline & & 19740 & 1050 & 1793 & 798 & 846 \\
\hline & & 920 & 6304 & 230 & 141 & 129 \\
\hline & & 1041 & 73 & 954 & 51 & 124 \\
\hline & & 590 & 239 & 46 & 4004 & 135 \\
\hline & & 373 & 74 & 35 & 33 & 836 \\
\hline \multicolumn{3}{|c|}{ Producer's accuracy } & \multicolumn{4}{|c|}{ User's accuracy } \\
\hline \multirow{5}{*}{\multicolumn{2}{|c|}{$\begin{array}{r}\text { Soil: } \\
\text { Vegetation: } \\
\text { Road: } \\
\text { Water: } \\
\text { Habitation: }\end{array}$}} & 87.098 & \multicolumn{3}{|c|}{ Soil: $\quad 81.479$} & \\
\hline & & 81.447 & \multirow{4}{*}{$\begin{array}{r}\text { Vegetation: } \\
\text { Road: } \\
\text { Water: } \\
\text { Habitation: }\end{array}$} & \multicolumn{2}{|c|}{81.616} & \\
\hline & & 31.197 & & \multicolumn{2}{|c|}{42.532} & \\
\hline & & 79.650 & & \multicolumn{2}{|c|}{79.856} & \\
\hline & & 40.386 & & & & \\
\hline \multicolumn{6}{|c|}{ Overall accuracy: $78.498 \%$} & \\
\hline
\end{tabular}

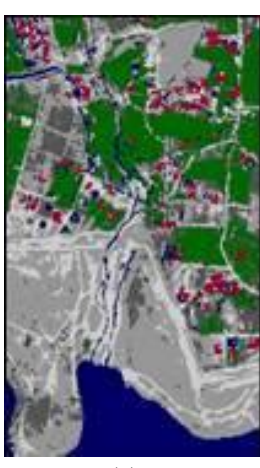

(a)

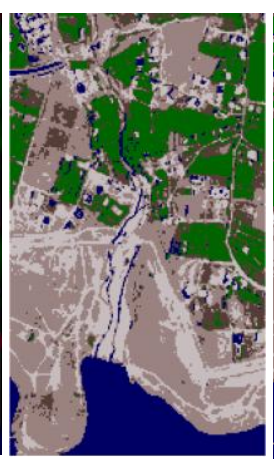

(b)

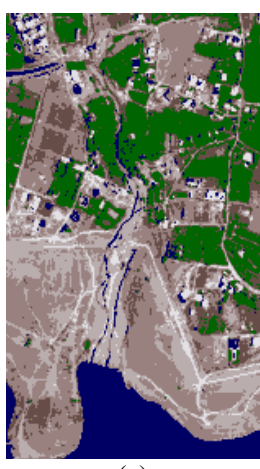

(c)
Fig. 9. clustered image using (a) GA, (b)K-means(5 clusters), (c) K-means clustering ( 5 clusters)

TABLE VI: COMPARISON THE PERFORMANCE OF ICA, K-MEANS(6-CLASS) AND GA INTEMRS OF ACCURACY

\begin{tabular}{|c|c|c|c|c|c|c|c|c|c|c|c|}
\hline \multirow{2}{*}{\begin{tabular}{|l|} 
Clusterin \\
g \\
algorithm
\end{tabular}} & \multicolumn{5}{|c|}{ User's accuracy } & \multicolumn{5}{|c|}{ Producer's accuracy } & \multirow{2}{*}{$\begin{array}{l}\text { overall } \\
\text { accurac } \\
\mathbf{y}\end{array}$} \\
\hline & - & 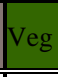 & $\begin{array}{l}\text { Roa } \\
\text { d }\end{array}$ & & $\mathrm{Hab}$ & $\overline{\text { Soil }}$ & Veg & $\begin{array}{l}\text { Roa } \\
\text { d } \\
\end{array}$ & & $\mathrm{Hab}$ & \\
\hline & 81.5 & 1.6 & 42.5 & 79.8 & 40.4 & $8 \% .1$ & 81.4 & 31.2 & 79.6 & 40.4 & \\
\hline & 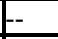 & & +- & - & -- & -- & - & - & - & $f$ & \\
\hline A & 80.3 & 81.1 & 27 & 81.7 & 53.1 & 76.6 & 81.4 & 49.8 & 79.6 & 39.2 & 74.03 \\
\hline
\end{tabular}

\section{CONCLUSION}

The paper employed the imperialist competitive algorithm which is a numerical optimization algorithm inspired from socio-political concepts in automatic image clustering. This algorithm was used to find the optimal cluster numbers and best cluster centers. The effectiveness of the combination of ICA and two stages fuzzy cluster analysis is demonstrated by the example of pan-sharpened IKONOS imagery and 4 artificial data sets with different overlapping properties. By using the proposed method, it was shown that the fuzzy hierarchical ICA based algorithm was able to determine the number of optimal clusters in data sets with various 
properties and clustering them with a high accuracy in comparison with two of most common clustering algorithms. However, the effect of scene type on automated image clustering needs to be further investigated.

\section{REFERENCES}

[1] S. Guha, R. Rastogu, and K. shim, "An efficient clustering for large data base", Proceeding of the ACM SIGMOD International conference on Management of Data, pp.73-84, 1998.

[2] T. M. Lillesand and R. W. Kiefer, "Remote sensing and image interpretation", John Wiley and Sons, New York, 724p, 2000.

[3] J. Zhang and G. M. Foody, "Full fuzzy supervised classification of sub-urban land cover from remotely sensed imagery: statistical neural network approaches", International Journal of Remote Sensing, Vol. 22, pp. 615, 2001.

[4] F. Rothlauf, "Representations for genentic and evolutionary algorithms", Springer, Netherlands, 314p, 2006.

[5] Gautam Garai, B.B.Chaudhuri, "A novel genetic algorithm for automatic clustering", Pattern Recognition Letters. Vol. 25, pp.173-187, 2004.

[6] E. Atashpaz-Gargari and C. Lucas, "Imperialist competitive algorithm: An algorithm for optimization inspired by imperialistic competition", IEEE Congress on Evolutionary Computation, Singapore, 2007.

[7] R. Rajabioun, F. Hashemzadeh, E. atashpaz-Gargari, B. Mesgari, and F. R. Salmasi, "Decentralized PID controller design for a MIMO evaporator based on colonial competitive algorithm", in 17th IFAC World congress, Seoul, Korea, July 6-11, 2008.

[8] E. Atashpaz-Gargari, F. Hashemzadeh, and C. Lucas, "Evolutionary design of PID controller for a MIMO distillation column using colonial competitive algorithm", international journal of intellegent computing and cybernetics, vol. 1, no. 3, pp. 337-355, 2008.

[9] Biabangard-Oskouyi, E. Atashpaz-Gargari, N. Soltani, and C. Lucas. "Application of imperialist competitive algorithm for materials property characterization from sharp indentation test", International Journal of Engineering Simulation, 2008.
[10] A. Khhabbazi, E. atashpaz, and caro lucas, "Imperialist competitive algorithm for minimum bit error rate beamforming", internatilonal journal of Bio-Inspired computation, vol. 1, pp.125-133, 2009.

[11] E. Atashpaz-Gargari and C.Lucas, "Colonial aompetitive algorithm as a tool for nash equilibrium point achievement", Lecture notes in computer science, Springer Berlin, vol. 5073, pp.680 695, 2008.

[12] E. J. Hajic and L. R. Tinney, "Analysis of visible and thermal Infrared data", Manual of RS, vol. 2.2nd ed., American Society of Photogrammetry, falls church, VA.1983.

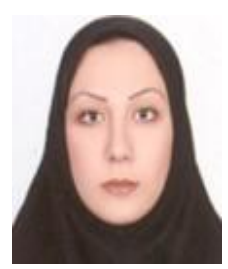

S. Karami was born in Tehran, Iran, March 20, 1982. She received her BS degree in Electrical Engineering from Amirkabir University of Technology (Tehran Polytechnic), Tehran, Iran in 2006 and the MS degree in Electrical engineering from Iran University of Science and Technology (IUST), Tehran, Iran in 2009. Her research interests are image processing, machine vision, pattern recognition and evolutionary computation. M.s Karami is the corresponding author and can be contacted at:Karami_samaneh@yahoo.com

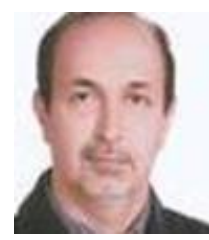

S. B. Shokouhi received his B.S. and M.S. degrees in Electronics in 1986 and 1989, both from the Department of Electrical Engineering of Iran University of Science and Technology (IUST). He received his Ph.D. on Medical Imaging and Image Processing in 1999 from the School of Electronic and Electrical Engineering, University of Bath, England. $\mathrm{He}$ is currently an assistant professor of Electrical Engineering Department at IUST. His research interests include Machine Vision Algorithms and Hardware Implementations, Pattern Recognition, and Intelligent Systems Design. Dr. Shokouhi is member of some international scientific societies such as IEEE, IEICE and also Iranian Machine Vision and Image Processing (MVIP). 